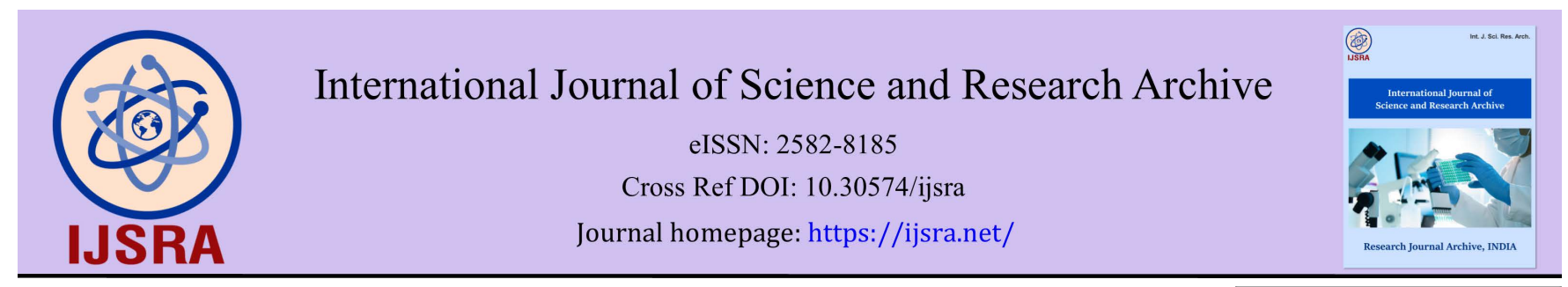

(REVIEW ARTICLE)

\title{
The same old story: Workplace violence towards healthcare providers during COVID- 19 pandemic
}

\author{
Ali Kemal Erenler ${ }^{1, *}$, Seval Komut ${ }^{1}$ and Ahmet Baydin ${ }^{2}$ \\ ${ }^{1}$ Emergency Medicine Hitit University, School of Medicine, Department of Emergency Medicine, Corum, Turkey. \\ ${ }^{2}$ Emergency Medicine Ondokuzmayis University, School of Medicine, Department of Emergency Medicine, Samsun, Turkey.
}

International Journal of Science and Research Archive, 2021, 03(01), 001-004

Publication history: Received on 23 May 2021; revised on 01 July 2021; accepted on 03 July 2021

Article DOI: https://doi.org/10.30574/ijsra.2021.3.1.0087

\begin{abstract}
Workplace violence (WPV) is a growing public health problem worldwide affecting physical and mental health of healthcare providers. It has many deterious consequences such as anxiety, burnout and intention to leave the job. With the pandemic, it is assumed that the incidence of workplace violence tends to increase. Particularly, misinformation about the nature of the disease create prejudice against staff working in healthcare facilities. There are several measures to be taken for prevention of physical and mental health of healthcare providers. A "zero-tolerance policy against violence" should be implemented. Doctors and nurses should be encouraged to report incidents. Social support should be provided for the personnel.

In this review, our aim was to clarify if there is an increase in the incidence of WPV against healthcare providers in the pandemic process. We also aimed to make recommendations about measures that must be taken to prevent healthcare providers from detrimental effects of WPV.
\end{abstract}

Keywords: Workplace violence; COVID-19; Healthcare providers; Workplace violence

\section{Introduction}

Workplace violence (WPV) is a growing problem worldwide, particularly for those in the healthcare facilities. Its frequency is on a rise which makes it a public health problem. Characteristics of WPV are similar in different parts of the World with different sociocultural and economic status. The definition of WPV is "Incidents in which an employee is abused, sexually harassed or assaulted in circumstances related to their work, involving an explicit or implicit challenge to their safety, well-being or health". The reasons for violence were reported to be unmet expectations by patients or their family members and friends (1). It is also known that delays in the hospital setting due to overcrowding and other reasons may also result in WPV against healthcare providers (2).

Psychological effects of this unwanted situation may arise as bothersome memories, super alertness and feelings of avoidance and futility (1).

In this review, we aimed to investigate the effect of COVID-19 pandemic on WPV towards healthcare providers and make recommendations to reduce WPV.

\footnotetext{
${ }^{*}$ Corresponding author: Ali Kemal Erenler

Associate Professor in Emergency Medicine Hitit University, School of Medicine, Department of Emergency Medicine, Çorum, Turkey. Copyright (C) 2021 Author(s) retain the copyright of this article. This article is published under the terms of the Creative Commons Attribution Liscense 4.0.
} 


\section{Material and methods}

We performed a narrative review of the literature by entering the keywords "COVID-19" and "workplace violence" to the scientific database, Pubmed ${ }^{\odot}$. A total of 32 articles were obtained. These articles (original contributions, reviews, letters, case reports, case series, etc.) were evaluated in terms of accuracy to the scope of the review. Those with irrelevant content, written in another language than English, without full-text or an explanatory abstract were excluded. Finally, a total of 18 publications were included into the study. The publications were discussed among the authors and written by consensus.

\section{Discussion}

Healthcare workers are essential component of every healthcare system. In the pandemic era, they fight on the frontline and are exposed to life-threatening hazards. These hazards may be listed as coronavirus exposure, long working hours, psychological stress, fatigue and workplace-related burnout. Healthcare workers also face the stress and concern about transmitting the virus to their family members. All these parameters result in a constant sense of intense fear, stigmatization, and ostracism when treating patients with COVID-19 (3).

It is also known that anxiety of the hospital staff is associated with social support, working at designated hospitals, longer working hours, discrimination experience, and medical WPV (4).

A study in Belgium revealed that violence, burnout and COVID-19 were the leading factors creating anxiety in healthcare workers. It was also reported that risk of violence and burnout has increased in the time of COVID-19 (5).

In China, it was documented that healthcare providers have faced difficult ethical/moral decisions given the enormous influx of patients with life-threatening infections and limitations in medical supplies during the pandemic. In addition, increasing numbers of violence against healthcare staff is being reported, globally. A mistrust towards the doctors created by conspiracy theories and a fear of catching the virus from healthcare providers may emerge as a violence to healthcare providers $(6,7)$. In a study, the incidence of WPV targeting healthcare providers was found to be18.5\% following the first two months of the COVID-19 pandemic (8). However, the real incidence may be much higher than that since many of the incidents are not being reported (1).

Limited access to medical care, overcrowding, prolonged length of stay in the hospital and limited communication with the healthcare staff results in dissatisfaction of patients and their relatives. These factors are the main reasons for workplace violence towards the staff. In addition, frontline clinicians face great pressure and overwhelming workload during the COVID-19 pandemic exacerbating their emotional disturbances, and affecting communication with patients and families (8).

In a study, it was reported that the prevalence of WPV was 18.5\% during COVID-19 outbreak. When types of violence were investigated, $15.8 \%$ reported verbal abuse and/or threats and 8.4\% reported physical assault. As before the pandemic, male health professionals were found to experience WPV more frequently during the pandemic (9). In another study on mental well-being of healthcare professionals, the prevalence of anxiety, depression, and stress among nurses was 69.6\%, 55.3\%, and 20.5\%, respectively. Risks for developing anxiety were lack of a standart guideline, fear of infecting family, and having chronic diseases (10).

In a study, $8.4 \%$ of the nurses stated that they were exposed to physical violence, $57.8 \%$ to verbal violence, $0.8 \%$ to sexual violence and $61.6 \%$ to mobbing during the COVID-19 pandemic. Additionally, 52.1\% of them thought of quitting their job (11).

In Hong Kong, a study revealed that workplace violence against clinicians was signifcantly related to anxiety (12). By the negative effects of workplace violence along with the pandemic stress, healthcare providers are also under the risk for post-traumatic stress disorder (PTSD). The prevalance of PTSD was shown to increase among staff after the pandemic (13). In a systematic review before the COVID-19 pandemic, it was reported that the prevalence of turnover intention among Chinese primary health workers reached as high as $30.4 \%$ (14).

In Sudan, doctors stated that they would go on strike after violenct acts against them. Hence, a police force to protect facilities was created. India declared that violent acts against healthcare professionals would be punished by up to 7 years' imprisonment thanks to the efforts of Indiam Medical Association (15). 
It is obvious that well-being of healthcare workers is essential to prevent serious consequences for patients and a possible collapse in our health systems (16).

The acts of violence against health-care workers serving during pandemic, as well as the post pandemic period, should be considered non-bailable offences, and the punishment for the offender should be imprisonment (17). Offenders must know that the government has a zeo-tolerance policy for violence against healthcare providers.

\section{Conclusion}

Widespread misinformation about COVID-19 accompanied with fear and anxiety in the community results in violence against healthcare professionals.

Since violence has "no excuse", hospital administrators and policy makers should adopt "zero-tolerance policy" for violence.

Violence against health-care personnel must be prevented and condemned. Preventions for violence must be discussed and determined with the partnerships of the with civil society, community-based groups, and media organizations. The incidence and types of attacks must be clearly determined and staff must be encouraged for reporting.

According to strict measures should be taken to stop violence against healthcare professionals. First of all, offenders must be held accountable for their actions and prosecuted adequately.

Second, detrimental impacts of WPV resulting in burnout and turnover intention should be decreased by enhancing social support.

Also, creating a safe working environment through logistical support, nondiscrimination, an arrangement of adequate rest would appear to alleviate the psychological pressure on the personnel.

Governments, policy makers and hospital administrations play an essential role in preventing physical and mental health of healthcare providers in the pandemic process.

\section{Compliance with ethical standards}

\section{Acknowledgments}

None to declare.

\section{Disclosure of conflict of interest}

The authors have no conflict of interests.

\section{References}

[1] Baydin A, Erenler AK. Workplace violence in emergency department and its effects on emergency staff. Int J Emerg Ment Health. 2014; 16(2): 288-290.

[2] Erenler AK, Akbulut S, Guzel M, et al. Reasons for Overcrowding in the Emergency Department: Experiences and Suggestions of an Education and Research Hospital. Turk J Emerg Med. 2016; 14(2): 59-63.

[3] Chew NWS, Lee GKH, Tan BYQ, et al. A multinational, multicentre study on the psychological outcomes and associated physical symptoms amongst healthcare workers during COVID-19 outbreak. Brain Behav Immun. 2020; S0889-1591: 30523-30527.

[4] Yang Y, Lu L, Chen T, et al. Healthcare Worker's Mental Health and Their Associated Predictors During the Epidemic Peak of COVID-19. Psychol Res Behav Manag. 2021; 14: 221-231.

[5] Somville F, Vanspringel G, De Cauwer H, Franck E, Van Bogaert P. Work stress-related problems in physicians in the time of COVID-19 [published online ahead of print, 2021 Jan 8]. Int J Occup Med Environ Health. 2021; 128524. 
[6] Zhizhong W, Koenig HG, Yan T, et al. Psychometric properties of the moral injury symptom scale among Chinese health professionals during the COVID-19 pandemic. BMC Psychiatry. 2020; 20(1): 556.

[7] Bhatti OA, Rauf H, Aziz N, Martins RS, Khan JA. Violence against Healthcare Workers during the COVID-19 Pandemic: A Review of Incidents from a Lower-Middle-Income Country. Ann Glob Health. 2021; 87(1): 41.

[8] Yang Y, Li Y, An Y, et al. Workplace Violence Against Chinese Frontline Clinicians During the COVID-19 Pandemic and Its Associations With Demographic and Clinical Characteristics and Quality of Life: A Structural Equation Modeling Investigation. Front Psychiatry. 2021; 12: 649989.

[9] Xie XM, Zhao YJ, An FR, et al. Workplace violence and its association with quality of life among mental health professionals in China during the COVID-19 pandemic. J Psychiatr Res. 2021; 135: 289-293.

[10] Mekonen E, Shetie B, Muluneh N. The Psychological Impact of COVID-19 Outbreak on Nurses Working in the Northwest of Amhara Regional State Referral Hospitals, Northwest Ethiopia. Psychol Res Behav Manag. 2021; 13: $1353-1364$.

[11] Özkan Şat S, Akbaş P, Yaman Sözbir Ş. Nurses' exposure to violence and their professional commitment during the COVID-19 pandemic. J Clin Nurs. 2021; 30(13-14): 2036-2047.

[12] Cheung T, Yip PS. Workplace violence towards nurses in Hong Kong: prevalence and correlates. BMC Public Health. 2017; 17 (1): 196.

[13] Marco CA, Larkin GL, Feeser VR, Monti JE, Vearrier L; ACEP Ethics Committee. Post-traumatic stress and stress disorders during the COVID-19 pandemic: Survey of emergency physicians. J Am Coll Emerg Physicians Open. 2020; 1(6): 1594-1601.

[14] Yang Y, Wang P, Kelifa MO, et al. How workplace violence correlates turnover intention among Chinese health care workers in COVID-19 context: The mediating role of perceived social support and mental health [published online ahead of print, 2021 Apr 1]. J Nurs Manag. 2021.

[15] Devi S. COVID-19 exacerbates violence against health workers. Lancet. 2020; 396(10252): 658.

[16] Rodríguez-Bolaños R, Cartujano-Barrera F, Cartujano B, Flores YN, Cupertino AP, Gallegos-Carrillo K. The Urgent Need to Address Violence Against Health Workers During the COVID-19 Pandemic. Med Care. 2020; 58(7): 663.

[17] Padubidri JR, Saran J, Manoj MA, Menezes RG. Amendment to the law to curb violence against doctors during the coronavirus disease 2019 crisis in India. Med Sci Law. 2021; 61(1): 74-75. 